\title{
Technological Process of Increasing the Density of Elements in a Compact Transimpedance Amplifier based on Heterostructures, Porosity and Missmatch Induced Stress of Materials
}

\author{
E.L. Pankratov \\ ${ }^{1}$ Nizhny Novgorod State University, 23 Gagarin avenue, Nizhny Novgorod, 603950, Russia \\ ${ }^{2}$ Nizhny Novgorod State Technical University, 24 Minin Street, Nizhny Novgorod, 603950, Russia
}

\begin{abstract}
In this paper, we introduce an approach to increase the density of field-effect transistors framework a compact transimpedance amplifier. In the present theoretical framework, the approach we consider in the manufacturing the inverter in heterostructure with specific configuration. Several required areas of the heterostructure should be doped by diffusion (alternatively they could be doped by ion implantation). After the considered doping dopant and radiation defects should by annealed framework optimized scheme. In this study, we also consider an approach to decrease the values of mismatch-induced stress in the considered heterostructure. An analytical approach is used to analyze the mass and heat transport in heterostructure during the manufacturing of integrated circuits with account of mismatchinduced stress.
\end{abstract}

KEYWORDS: Analytical approach, Transimpedance amplifier; optimization, accounting of mismatch induced stress and porosity of materials.

https://doi.org/10.29294/IJASE.8.3.2022.2242-2251

CC2022 Mahendrapublications.com, All rights reserved

\section{INTRODUCTION}

In the present days, several problems of the solid state electronics (such as increasing of performance, reliability and density of elements of integrated circuits: diodes, field-effect and bipolar transistors) are intensively solving [1-6]. To increase the performance of the devices, it is attracted an interest in the determination of materials with higher values of charge carriers mobility [7-10]. One way to decrease the dimensions of elements of integrated circuits is by manufacturing them in thin film heterostructures $[3-5,11]$. In this case, it is possible to use inhomogeneity of heterostructure and necessary optimization of doping of electronic materials [12] and to develop an epitaxial technology to improve these materials (including the analysis of mismatch induced stress) [13-15]. An alternative approaches to increase the dimensions of integrated circuits are using of laser and microwave types of annealing [16-18].

Framework, in this paper we introduce an approach to manufacture field-effect transistors. This approach gives a possibility to decrease their dimensions with increasing their density framework a compact transimpedance amplifier. We also consider the possibility to decrease mismatch-induced stress to decrease quantity of defects, generated due to the stress. Also, we consider a heterostructure which consist of a substrate and an epitaxial layer (see Fig. 1), and a buffer layer between the substrate and the epitaxial layer. The epitaxial layer includes itself several sections, which were manufactured by using another materials. These sections have been doped by diffusion or ion implantation to manufacture the required types of conductivity ( $p$ or $n$ ). These areas became sources, drains and gates (see Fig. 1). After doping, it is required to annealing of dopant and/or radiation defects. The objective of the present paper is to analysis the redistribution of dopant and radiation defects to determine under conditions, which correspond to decreasing of elements of the considered voltage reference and at the same time to increase their density. At the same time, we consider a possibility to decrease mismatch-induced stress.

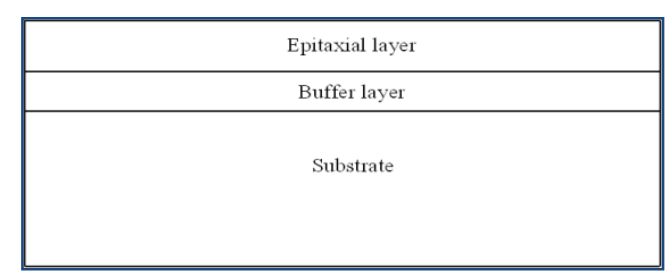

Fig. $1 b$ Heterostructure with a substrate, epitaxial layers and buffer layer (view from side)

\begin{tabular}{lcc}
\hline & & \\
& $*$ & \\
Received $: 15.12 .2021$ & Accepted: 27.01 .2022 & Published on: 22.02 .2022 \\
\hline
\end{tabular}




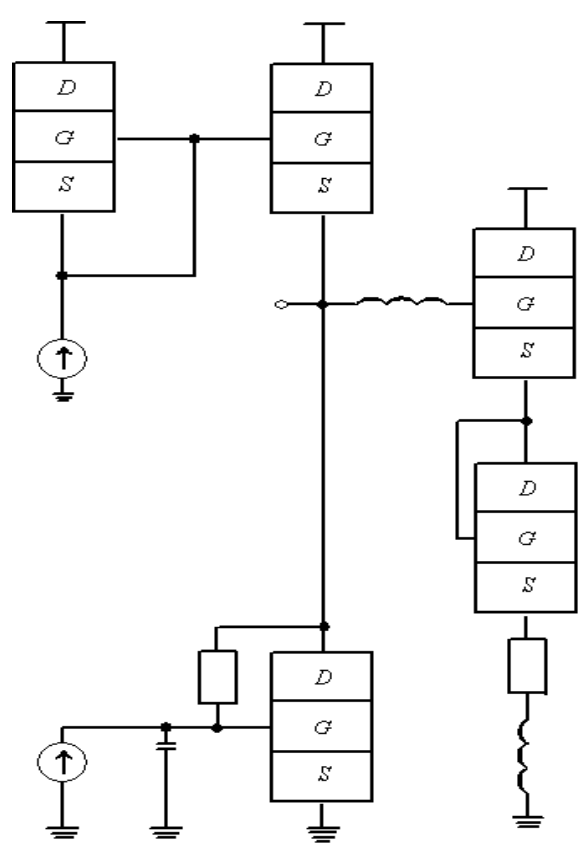

Fig. 1a. Structure of the considered amplifier [19]

\section{METHOD OF SOLUTION}

To solve our aim, we determine and analyzed the spatio-temporal distribution of concentration of dopant in the considered heterostructure. We determine the distribution by solving the second Fick's law in the following form [1,20-23]

$$
\begin{aligned}
& \frac{\partial C(x, y, z, t)}{\partial t}=\frac{\partial}{\partial x}\left[D \frac{\partial C(x, y, z, t)}{\partial x}\right]+\frac{\partial}{\partial y}\left[D \frac{\partial C(x, y, z, t)}{\partial y}\right]+\frac{\partial}{\partial z}\left[D \frac{\partial C(x, y, z, t)}{\partial z}\right]+ \\
& +\Omega \frac{\partial}{\partial x}\left[\frac{D_{S}}{k T} \nabla_{S} \mu_{1}(x, y, z, t) \int_{0}^{L_{z}} C(x, y, W, t) d W\right]+ \\
& +\Omega \frac{\partial}{\partial y}\left[\frac{D_{S}}{k T} \nabla_{S} \mu_{1}(x, y, z, t) \int_{0}^{L_{z}} C(x, y, W, t) d W\right]+(1) \\
& +\frac{\partial}{\partial x}\left[\frac{D_{C S}}{\bar{V} k T} \frac{\partial \mu_{2}(x, y, z, t)}{\partial x}\right]+\frac{\partial}{\partial y}\left[\frac{D_{C S}}{\bar{V} k T} \frac{\partial \mu_{2}(x, y, z, t)}{\partial y}\right]+\frac{\partial}{\partial z}\left[\frac{D_{C S}}{\bar{V} k T} \frac{\partial \mu_{2}(x, y, z, t)}{\partial z}\right]
\end{aligned}
$$

with boundary and initial conditions

$$
\begin{gathered}
\left.\frac{\partial C(x, y, z, t)}{\partial x}\right|_{x=0}=0,\left.\frac{\partial C(x, y, z, t)}{\partial x}\right|_{x=L_{x}}=0,\left.\frac{\partial C(x, y, z, t)}{\partial y}\right|_{y=0}=0, C(x, y, z, 0)=f_{C}(x, y, z), \\
\left.\frac{\partial C(x, y, z, t)}{\partial y}\right|_{x=L_{y}}=0,\left.\frac{\partial C(x, y, z, t)}{\partial z}\right|_{z=0}=0,\left.\frac{\partial C(x, y, z, t)}{\partial z}\right|_{x=L_{z}}=0 .
\end{gathered}
$$

Here $C(x, y, z, t)$ is the spatio-temporal distribution of dopant; $\nabla_{s}$ is the symbol of surficial gradient; of concentration of dopant; $\Omega$ is the atomic volume

\section{Pankratov}


$\int_{0}^{L_{z}} C(x, y, z, t) d z$ is the surficial concentration of dopant on interface between layers of heterostructure (in this situation we assume, that $\mathrm{Z}$-axis is perpendicular to interface between layers of heterostructure); $\mu_{1}(x, y, z, t)$ and $\mu_{2}(x, y, z, t)$ are the chemical potential due to the presence of mismatch-induced stress and porosity of material;
$D$ and $D_{S}$ are the coefficients of volumetric and surficial diffusions. Values of dopant diffusions coefficients depends on properties of materials of heterostructure, speed of heating and cooling of materials during annealing and spatio-temporal distribution of concentration of dopant. Dependences of dopant diffusions coefficients on parameters could be approximated by the following relations [24-26]

$$
\begin{gathered}
D_{C}=D_{L}(x, y, z, T)\left[1+\xi \frac{C^{\gamma}(x, y, z, t)}{P^{\gamma}(x, y, z, T)}\right]\left[1+\varsigma_{1} \frac{V(x, y, z, t)}{V^{*}}+\varsigma_{2} \frac{V^{2}(x, y, z, t)}{\left(V^{*}\right)^{2}}\right], \\
D_{S}=D_{S L}(x, y, z, T)\left[1+\xi_{S} \frac{C^{\gamma}(x, y, z, t)}{P^{\gamma}(x, y, z, T)}\right]\left[1+\varsigma_{1} \frac{V(x, y, z, t)}{V^{*}}+\varsigma_{2} \frac{V^{2}(x, y, z, t)}{\left(V^{*}\right)^{2}}\right] .
\end{gathered}
$$

Here $D_{L}(x, y, z, T)$ and $D_{L S}(x, y, z, T)$ are the spatial (due to accounting all layers of heterostruicture) and temperature (due to Arrhenius law) dependences of dopant diffusion coefficients; $T$ is the temperature of annealing; $P(x, y, z, T)$ is the limit of solubility of dopant; parameter $\gamma$ depends on properties of materials and could be integer in the following interval $\gamma \in[1,3][24] ; V(x, y, z, t)$ is the spatio-temporal distribution of concentration of radiation vacancies; $V^{*}$ is the equilibrium distribution of vacancies. Concentrational dependence of dopant diffusion coefficient has been described in details in [24]. Spatio-temporal distributions of concentration of point radiation defects have been determined by solving the following system of equations $[20-23,25,26]$

$$
\begin{gathered}
\frac{\partial \rho(x, y, z, t)}{\partial t}=\frac{\partial}{\partial x}\left[D_{\rho}(x, y, z, T) \frac{\partial \rho(x, y, z, t)}{\partial x}\right]+\frac{\partial}{\partial y}\left[D_{\rho}(x, y, z, T) \frac{\partial \rho(x, y, z, t)}{\partial y}\right]+ \\
+\frac{\partial}{\partial z}\left[D_{\rho}(x, y, z, T) \frac{\partial \rho(x, y, z, t)}{\partial z}\right]-k_{\rho, \rho}(x, y, z, T) \rho^{2}(x, y, z, t)-k_{I, V}(x, y, z, T) \times \\
\quad \times I(x, y, z, t) V(x, y, z, t)+\Omega \frac{\partial}{\partial x}\left[\frac{D_{\rho S}}{k T} \nabla_{S} \mu(x, y, z, t) \int_{0}^{L_{z}} \rho(x, y, W, t) d W\right]+ \\
+\Omega \frac{\partial}{\partial y}\left[\frac{D_{\rho S}}{k T} \nabla_{S} \mu(x, y, z, t) \int_{0}^{L_{z}} \rho(x, y, W, t) d W\right]+\frac{\partial}{\partial x}\left[\frac{D_{\rho S}}{\bar{V} k T} \frac{\partial \mu_{2}(x, y, z, t)}{\partial x}\right]+ \\
+\frac{\partial}{\partial y}\left[\frac{D_{\rho S}}{\bar{V} k T} \frac{\partial \mu_{2}(x, y, z, t)}{\partial y}\right]+\frac{\partial}{\partial z}\left[\frac{D_{\rho S}}{\bar{V} k T} \frac{\partial \mu_{2}(x, y, z, t)}{\partial z}\right]
\end{gathered}
$$

with boundary and initial conditions

$$
\begin{aligned}
& \left.\frac{\partial \rho(x, y, z, t)}{\partial x}\right|_{x=0}=0,\left.\frac{\partial \rho(x, y, z, t)}{\partial x}\right|_{x=L_{x}}=0,\left.\frac{\partial \rho(x, y, z, t)}{\partial y}\right|_{y=0}=0 \\
& \left.\frac{\partial \rho(x, y, z, t)}{\partial y}\right|_{y=L_{y}}=0,\left.\frac{\partial \rho(x, y, z, t)}{\partial z}\right|_{z=0}=0,\left.\frac{\partial \rho(x, y, z, t)}{\partial z}\right|_{z=L_{z}}=0
\end{aligned}
$$

$$
\rho(x, y, z, 0)=f_{V}(x, y, z), V\left(x_{1}+V_{n} t, y_{1}+V_{n} t, z_{1}+V_{n} t, t\right)=V_{\infty}\left(1+2 \ell \omega / k T \sqrt{x_{1}^{2}+y_{1}^{2}+z_{1}^{2}}\right) \text {. }
$$

\section{Pankratov}


Here $\rho=I, V ; I(x, y, z, t)$ is the spatio-temporal distribution of concentration of radiation interstitials; $I^{*}$ is the equilibrium distribution of interstitials; $D_{I}(x, y, z, T), \quad D_{V}(x, y, z, T), D_{I S}(x, y, z, T)$, $D_{V S}(x, y, z, T)$ are the coefficients of volumetric and surficial diffusions of interstitials and vacancies, respectively; terms $V^{2}(x, y, z, t)$ and $I^{2}(x, y, z, t)$ correspond to generation of divacancies and diinterstitials, respectively (see, for example, [26] and appropriate references in this book); $k_{I, V}(x, y, z, T), \quad k_{I, I}(x, y, z, T)$ and $k_{V, V}(x, y, z, T)$ are the parameters of recombination of point radiation defects and generation of their complexes; $k$ is the
Boltzmann constant; $\omega=a^{3}, a$ is the interatomic distance; $\ell$ is the specific surface energy. To account porosity of buffer layers we assume, that porous are approximately cylindrical with average values $r=\sqrt{x_{1}^{2}+y_{1}^{2}}$ and $z_{1}$ before annealing [23]. With time small pores combine into large ones [27]. With time large pores became larger due to absorbing the vacancies and became more spherical [27]. Distribution of concentration of vacancies in heterostructure, existing due to porosity, could be determined by summing on all pores, i.e.

$$
V(x, y, z, t)=\sum_{i=0}^{l} \sum_{j=0}^{m} \sum_{k=0}^{n} V_{p}(x+i \alpha, y+j \beta, z+k \chi, t), R=\sqrt{x^{2}+y^{2}+z^{2}} .
$$

Here $\alpha, \beta$ and $\chi$ are the average distances between centers of pores in directions $x, y$ and $z ; l, m$ and $n$ are the quantity of pores inappropriate directions.

Spatio-temporal distributions of divacancies $\Phi_{V}(x, y, z, t)$ and diinterstitials $\Phi_{I}(x, y, z, t)$ could be determined by solving the following system of equations $[25,26]$

$$
\begin{aligned}
& \frac{\partial \Phi_{\rho}(x, y, z, t)}{\partial t}=\frac{\partial}{\partial x}\left[D_{\Phi_{\rho}}(x, y, z, T) \frac{\partial \Phi_{\rho}(x, y, z, t)}{\partial x}\right]+\frac{\partial}{\partial y}\left[D_{\Phi_{\rho}}(x, y, z, T) \frac{\partial \Phi_{\rho}(x, y, z, t)}{\partial y}\right]+ \\
& +\frac{\partial}{\partial z}\left[D_{\Phi_{\rho}}(x, y, z, T) \frac{\partial \Phi_{\rho}(x, y, z, t)}{\partial z}\right]+\Omega \frac{\partial}{\partial x}\left[\frac{D_{\Phi_{\rho} S}}{k T} \nabla_{s} \mu_{1}(x, y, z, t) \int_{0}^{L_{z}} \Phi_{\rho}(x, y, W, t) d W\right]+ \\
& +\Omega \frac{\partial}{\partial y}\left[\frac{D_{\Phi_{\rho} S}}{k T} \nabla_{S} \mu_{1}(x, y, z, t) \int_{0}^{L_{z}} \Phi_{\rho}(x, y, W, t) d W\right]+k_{\rho, \rho}(x, y, z, T) \rho^{2}(x, y, z, t)+ \\
& +\frac{\partial}{\partial x}\left[\frac{D_{\Phi_{\rho} S}}{\bar{V} k T} \frac{\partial \mu_{2}(x, y, z, t)}{\partial x}\right]+\frac{\partial}{\partial y}\left[\frac{D_{\Phi_{\rho} S}}{\bar{V} k T} \frac{\partial \mu_{2}(x, y, z, t)}{\partial y}\right]+\frac{\partial}{\partial z}\left[\frac{D_{\Phi_{\rho} S}}{\bar{V} k T} \frac{\partial \mu_{2}(x, y, z, t)}{\partial z}\right]+ \\
& +k k_{\rho}(x, y, z, T) \rho(x, y, z, t)
\end{aligned}
$$

with boundary and initial conditions

$$
\begin{gathered}
\left.\frac{\partial \Phi_{\rho}(x, y, z, t)}{\partial x}\right|_{x=0}=0,\left.\frac{\partial \Phi_{\rho}(x, y, z, t)}{\partial x}\right|_{x=L_{x}}=0,\left.\frac{\partial \Phi_{\rho}(x, y, z, t)}{\partial y}\right|_{y=0}=0, \\
\left.\frac{\partial \Phi_{\rho}(x, y, z, t)}{\partial y}\right|_{y=L_{y}}=0,\left.\frac{\partial \Phi_{\rho}(x, y, z, t)}{\partial z}\right|_{z=0}=0,\left.\frac{\partial \Phi_{\rho}(x, y, z, t)}{\partial z}\right|_{z=L_{z}}=0, \\
\Phi_{\rho}(x, y, z, 0)=f_{\Phi_{\rho}}(x, y, z) .
\end{gathered}
$$

Here $D_{\Phi I}(x, y, z, T), D_{\phi V}(x, y, z, T), D_{\Phi I S}(x, y, z, T)$ and $D_{\Phi V S}(x, y, z, T)$ are the coefficients of volumetric and surficial diffusions of complexes of radiation defects; $k_{I}(x, y, z, T)$ and $k_{v}(x, y, z, T)$ are the parameters of decay of complexes of radiation defects.

Chemical potential $\mu_{1}$ in Eq.(1) could be determine by the following relation [20]

\section{Pankratov}




$$
\mu_{1}=E(z) \Omega \sigma_{i j}\left[u_{i j}(x, y, z, t)+u_{j i}(x, y, z, t)\right] / 2,
$$

where $E(z)$ is the Young modulus, $\sigma_{i j}$ is the stress tensor; $u_{i j}=\frac{1}{2}\left(\frac{\partial u_{i}}{\partial x_{j}}+\frac{\partial u_{j}}{\partial x_{i}}\right)$ is the deformation tensor; $u_{i}, u_{j}$ are the components $u_{x}(x, y, z, t), u_{y}(x, y, z, t)$ and $u_{z}(x, y, z, t)$ of the displacement vector $\vec{u}(x, y, z, t) ; x_{i}, x_{j}$ are the coordinate $x, y, z$. The Eq. (3) could be transform to the following form

$$
\begin{aligned}
& \mu(x, y, z, t)=\left[\frac{\partial u_{i}(x, y, z, t)}{\partial x_{j}}+\frac{\partial u_{j}(x, y, z, t)}{\partial x_{i}}\right]\left\{\frac{1}{2}\left[\frac{\partial u_{i}(x, y, z, t)}{\partial x_{j}}+\frac{\partial u_{j}(x, y, z, t)}{\partial x_{i}}\right]-\right. \\
& \left.-\varepsilon_{0} \delta_{i j}+\frac{\sigma(z) \delta_{i j}}{1-2 \sigma(z)}\left[\frac{\partial u_{k}(x, y, z, t)}{\partial x_{k}}-3 \varepsilon_{0}\right]-K(z) \beta(z)\left[T(x, y, z, t)-T_{0}\right] \delta_{i j}\right\} \frac{\Omega}{2} E(z)
\end{aligned}
$$

where $\sigma$ is Poisson coefficient; $\varepsilon_{0}=\left(a_{s}-a_{E L}\right) / a_{E L}$ is the mismatch parameter; $a_{s}, a_{E L}$ are lattice distances of the substrate and the epitaxial layer; $K$ is the modulus of uniform compression; $\beta$ is the coefficient of thermal expansion; $T_{r}$ is the

$$
\left\{\begin{array}{l}
\rho(z) \frac{\partial^{2} u_{x}(x, y, z, t)}{\partial t^{2}}=\frac{\partial \sigma_{x x}(x, y, z, t)}{\partial x}+\frac{\partial \sigma_{x y}(x, y, z, t)}{\partial y}+\frac{\partial \sigma_{x z}(x, y, z, t)}{\partial z} \\
\rho(z) \frac{\partial^{2} u_{y}(x, y, z, t)}{\partial t^{2}}=\frac{\partial \sigma_{y x}(x, y, z, t)}{\partial x}+\frac{\partial \sigma_{y y}(x, y, z, t)}{\partial y}+\frac{\partial \sigma_{y z}(x, y, z, t)}{\partial z} \\
\rho(z) \frac{\partial^{2} u_{z}(x, y, z, t)}{\partial t^{2}}=\frac{\partial \sigma_{z x}(x, y, z, t)}{\partial x}+\frac{\partial \sigma_{z y}(x, y, z, t)}{\partial y}+\frac{\partial \sigma_{z z}(x, y, z, t)}{\partial z}
\end{array}\right.
$$

where

$$
\sigma_{i j}=\frac{E(z)}{2[1+\sigma(z)]}\left[\frac{\partial u_{i}(x, y, z, t)}{\partial x_{j}}+\frac{\partial u_{j}(x, y, z, t)}{\partial x_{i}}-\frac{\delta_{i j}}{3} \frac{\partial u_{k}(x, y, z, t)}{\partial x_{k}}\right]+K(z) \delta_{i j} \times
$$

$\times \frac{\partial u_{k}(x, y, z, t)}{\partial x_{k}}-\beta(z) K(z)\left[T(x, y, z, t)-T_{r}\right], \rho \quad(z)$ is the density of materials of heterostructure, $\delta_{i j}$ Is the Kronecker symbol. The conditions for the system in Eqs. (8) could be written in the form

$$
\begin{aligned}
& \frac{\partial \vec{u}(0, y, z, t)}{\partial x}=0 ; \frac{\partial \vec{u}\left(L_{x}, y, z, t\right)}{\partial x}=0 ; \frac{\partial \vec{u}(x, 0, z, t)}{\partial y}=0 ; \frac{\partial \vec{u}\left(x, L_{y}, z, t\right)}{\partial y}=0 ; \\
& \frac{\partial \vec{u}(x, y, 0, t)}{\partial z}=0 ; \frac{\partial \vec{u}\left(x, y, L_{z}, t\right)}{\partial z}=0 ; \vec{u}(x, y, z, 0)=\vec{u}_{0} ; \vec{u}(x, y, z, \infty)=\vec{u}_{0} .
\end{aligned}
$$

We calculate the spatio-temporal distributions of concentrations of dopant and radiation defects by solving the Eqs.(1), (3), (5) and (8) framework standard method of averaging of function corrections [28]. In this paper, we also determine the concentrations of dopant, concentrations of radiation defects and components of displacement vector by using the second-order approximation framework method of averaging of function corrections. This approximation is usually enough good approximation to make qualitative analysis and to obtain some quantitative results. All

\section{Pankratov}


obtained results have been checked by comparison with results of numerical simulations.

\section{DISCUSSION}

In this section, we analyzed the dynamics of redistributions of dopant and radiation defects during annealing, under the influence of mismatch-induced stress and modification of porosity. Typical distributions of concentrations of dopant in heterostructures are presented on Figs. 2 and 3 for diffusion and ion types of doping, respectively. These distributions have been calculated for the case, when the value of dopant diffusion coefficient in doped area is larger than in nearest areas. The Figures shown that the inhomogeneity of heterostructure gives us possibility to increase the compactness of concentrations of dopants and at the same time to increase homogeneity of dopant distribution in doped part of epitaxial layer. However, this approach of manufacturing of bipolar transistor it is necessary to optimize annealing of dopant and/or radiation defects. Reason of this optimization is following. If annealing time is small, the dopant did not achieve any interfaces between materials of heterostructure. In this situation one cannot find any modifications of distribution of concentration of dopant. If annealing time is large, distribution of concentration of dopant is too homogenous. We optimize annealing time framework recently introduces approach [29-37]. Framework this criterion we approximate real distribution of concentration of dopant by step-wise function (see Figs. 4 and 5). Farther we determine optimal values of annealing time by minimization of the following mean-squared error

$$
U=\frac{1}{L_{x} L_{y} L_{z}} \int_{0}^{L_{x}} \int_{0}^{L_{y}} \int_{0}^{L_{z}}[C(x, y, z, \Theta)-\psi(x, y, z)] d z d y d x
$$

Fig.2. Distributions of concentration of infused dopant in heterostructure from Fig. 1 in direction, which is perpendicular to interface between epitaxial layer substrate. Increasing of number of curve corresponds to increasing of difference between values of dopant diffusion coefficient in layers of heterostructure under condition, when value of dopant diffusion coefficient in epitaxial layer is larger, than value of dopant diffusion coefficient in substrate.

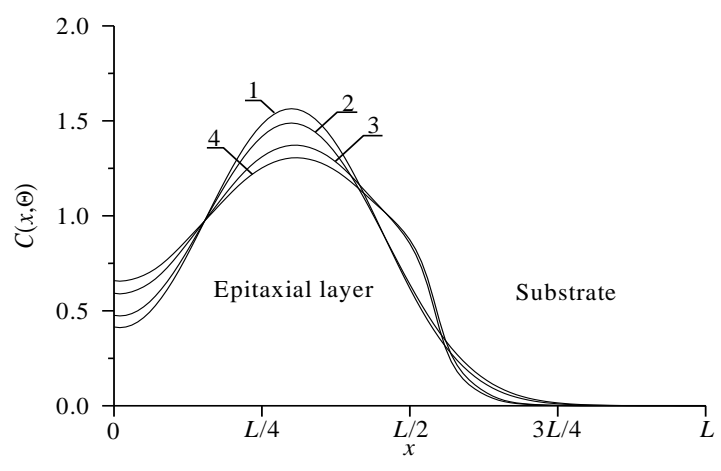

Fig.3. Distributions of concentration of implanted dopant in heterostructure from Fig. 1 in direction, which is perpendicular to interface between epitaxial layer substrate. Curves 1 and 3 corresponds to annealing time $\Theta=0.0048\left(L_{x}{ }^{2}+L_{y}{ }^{2}+L_{z}{ }^{2}\right) / D_{0}$. Curves 2 and 4 corresponds to annealing time $\Theta=0.0057\left(L_{x}{ }^{2}+L_{y}{ }^{2}+L_{z}{ }^{2}\right) / D_{0}$. Curves 1 and 2 corresponds to homogenous sample. Curves 3 and 4 corresponds to heterostructure under

\section{Pankratov}


condition, when value of dopant diffusion coefficient in epitaxial layer is larger, than value of dopant diffusion coefficient in substrate

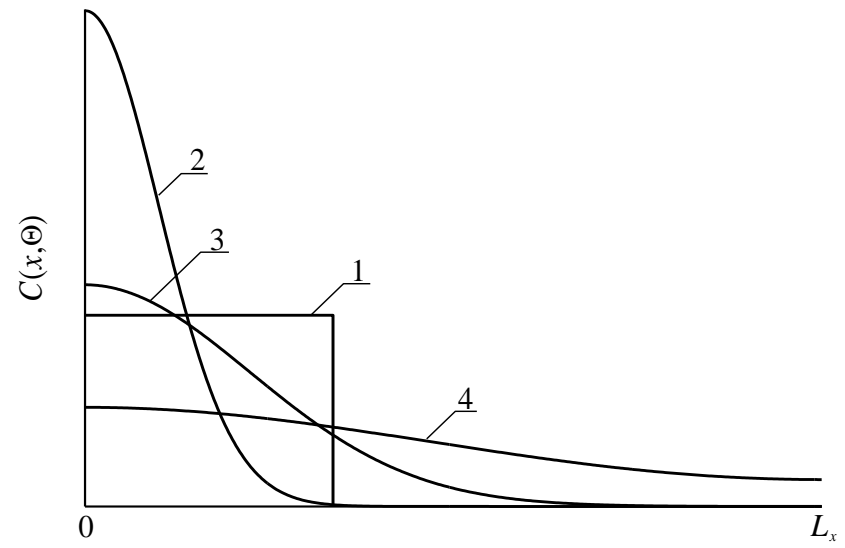

Fig. 4. Spatial distributions of dopant in heterostructure after dopant infusion. Curve 1 is idealized distribution of dopant. Curves 2-4 are real distributions of dopant for different values of annealing time. Increasing of number of curve corresponds to increasing of annealing time

where $\psi(x, y, z)$ is the approximation function. Dependences of optimal values of annealing time on parameters are presented on Figs. 6 and 7 for diffusion and ion types of doping, respectively. It should be noted, that it is necessary to anneal the radiation defects after ion implantation. One could find the spreading of concentration of distribution of dopant during annealing. In the ideal case distribution of dopant achieves appropriate interfaces between materials of heterostructure during annealing of radiation defects. If dopant did not achieve any interfaces during annealing of radiation defects, it is practicably to additionally anneal the dopant. In this situation optimal value of additional annealing time of implanted dopant is smaller, than annealing time of infused dopant.

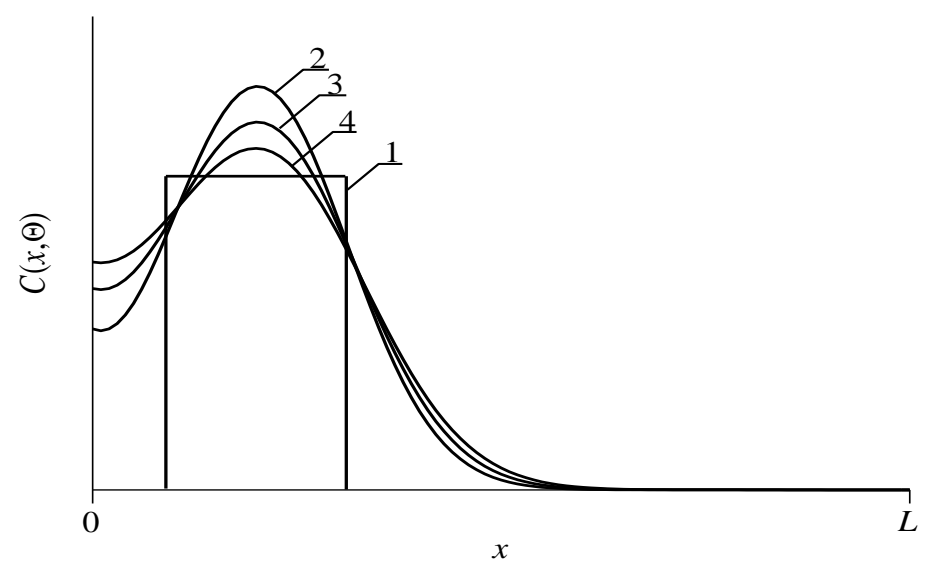

Fig. 5. Spatial distributions of dopant in heterostructure after ion implantation. Curve 1 is idealized distribution of dopant. Curves 2-4 are real distributions of dopant for different values of annealing time. Increasing of number of curve corresponds to increasing of annealing time

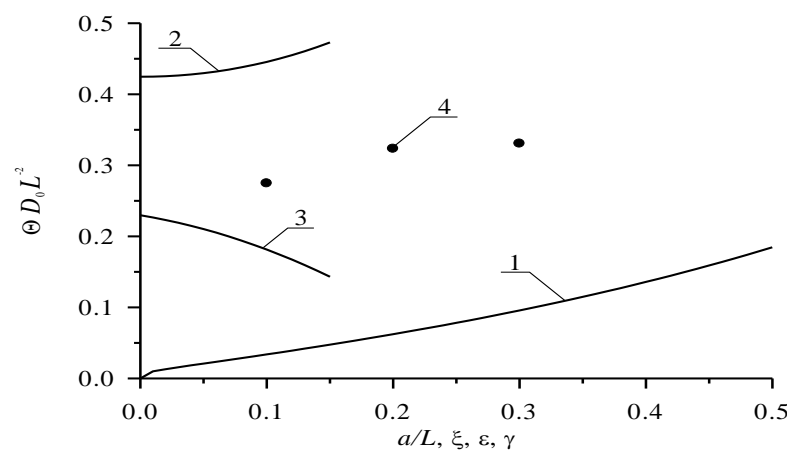

\section{Pankratov}


Fig.6. Dependences of dimensionless optimal annealing time for doping by diffusion, which have been obtained by minimization of mean-squared error, on several parameters. Curve 1 is the dependence of dimensionless optimal annealing time on the relation $a / L$ and $\xi=\gamma=0$ for equal to each other values of dopant diffusion coefficient in all parts of heterostructure. Curve 2 is the dependence of dimensionless optimal annealing time on value of parameter $\varepsilon$ for $a / L=1 / 2$ and $\xi=\gamma=0$. Curve 3 is the dependence of dimensionless optimal annealing time on value of parameter $\xi$ for $a / L=1 / 2$ and $\varepsilon=\gamma=0$. Curve 4 is the dependence of dimensionless optimal annealing time on value of parameter $\gamma$ for $a / L=1 / 2$ and $\varepsilon=\xi=0$

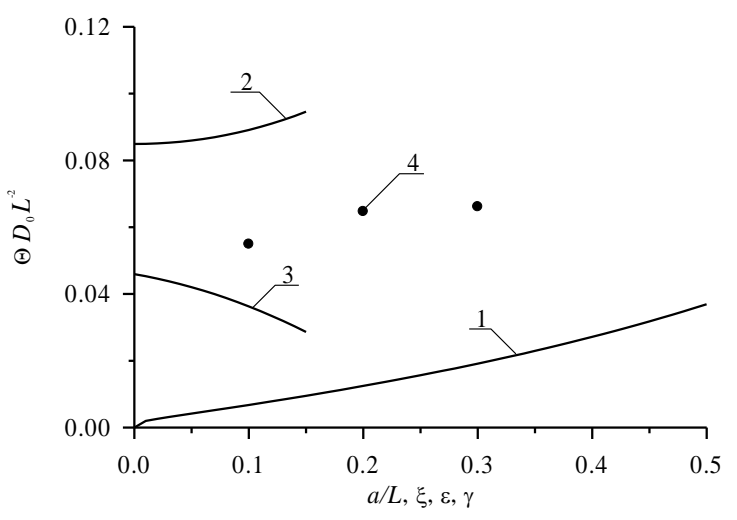

Fig.7. Dependences of dimensionless optimal annealing time for doping by ion implantation, which have been obtained by minimization of mean-squared error, on several parameters. Curve 1 is the dependence of dimensionless optimal annealing time on the relation $a / L$ and $\xi=\gamma=0$ for equal to each other values of dopant diffusion coefficient in all parts of heterostructure. Curve 2 is the dependence of dimensionless optimal annealing time on value of parameter $\varepsilon$ for $a / L=1 / 2$ and $\xi=\gamma=0$. Curve 3 is the dependence of dimensionless optimal annealing time on value of parameter $\xi$ for $a / L=1 / 2$ and $\varepsilon=\gamma=0$. Curve 4 is the dependence of dimensionless optimal annealing time on value of parameter $\gamma$ for $a / L=1 / 2$ and $\varepsilon=\xi=0$

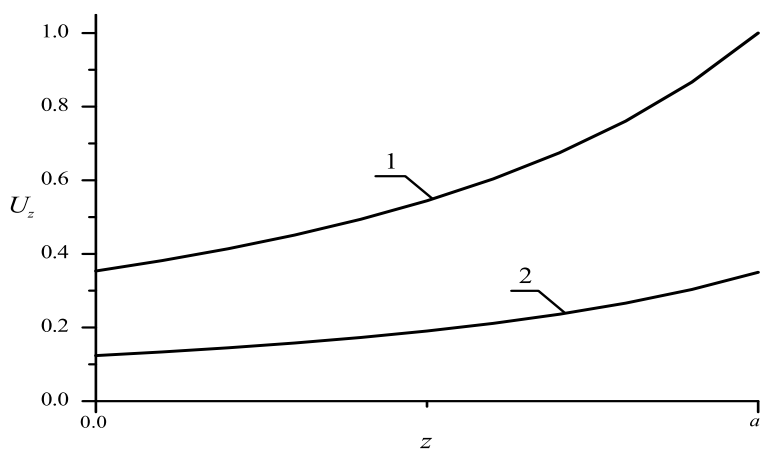

Fig. 8. Normalized dependences of component $u_{z}$ of displacement vector on coordinate $z$ for nonporous (curve 1) and porous (curve 2) epitaxial layers

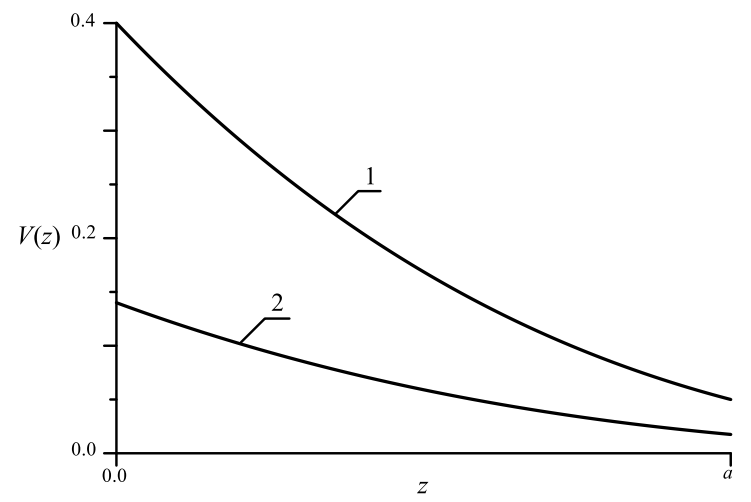

Fig. 9. Normalized dependences of vacancy concentrations on coordinate $z$ in unstressed (curve 1) and stressed (curve 2) epitaxial layers

\section{Pankratov}


Further, we analyzed influence of relaxation of mechanical stress on distribution of dopant in doped areas of heterostructure. Under following condition $\varepsilon_{0}<0$ one can find compression of distribution of concentration of dopant near interface between materials of heterostructure. Contrary (at $\varepsilon_{0}>0$ ) one can find spreading of distribution of concentration of dopant in this area. This changing of distribution of concentration of dopant could be at least partially compensated by using laser annealing [37]. This type of annealing gives us possibility to accelerate diffusion of dopant and another process in annealed area due to inhomogenous distribution of temperature and Arrhenius law. Accounting relaxation of mismatch-induced stress in heterostructure could leads to changing of optimal values of annealing time. At the same time modification of porosity gives us possibility to decrease value of mechanical stress. On the one hand mismatch-induced stress could be used to increase density of elements of integrated circuits. On the other hand could leads to generation dislocations of the discrepancy. Figs. 8 and 9 show distributions of concentration of vacancies in porous materials and component of displacement vector, which is perpendicular to interface between layers of heterostructure.

\section{CONCLUSION}

In this paper, we modeled the redistribution of infused and implanted dopants with account relaxation mismatch-induced stress during manufacturing field-effect heterotransistors framework a compact transimpedance amplifier. We also formulated the recommendations for optimization of annealing to decrease dimensions of transistors and to increase their density and the recommendations to decrease mismatch-induced stress. Analytical approach to model diffusion and ion types of doping with account concurrent changing of parameters in space and time has been introduced. At the same time the approach gives us possibility to take into account nonlinearity of considered processes.

\section{REFERENCES}

[1] Lachin, V.I., Savelov, N.S. Electronics (Rostov-on-Don: Phoenix, 2001).

[2] Polishscuk, A. 2004. Anadigm Programmable Analog ICs: The entire spectrum of analog electronics on a single chip. First meeting. Modern Electronics. 12. P. 8-11.

[3] Volovich, G. 2006. Modern chips UM3Ch class D manufactured by firm MPS. Modern Electronics. 2. P. 10-17.

[4] Kerentsev, A., Lanin, V. (2008). Constructive-technological features of
MOSFET-transistors. Power Electronics. 1. P. 34-38.

[5] Ageev, A.O., Belyaev, A.E., Boltovets, N.S., Ivanov, V.N., Konakova, R.V., Kudrik, Ya.Ya., Litvin, P.M., Milenin, V.V., Sachenko, A.V. 2009. Au-TiBx-n-6H-SiC Schottky barrier diodes: the features of current flow in rectifying and nonrectifying contacts. Semiconductors, 43 (7), 897-903.

[6] Tsai, J.-H., Chiu, Sh.-Y., Lour, W.-Sh., Guo, D.F. 2009. High-performance InGaP/GaAs pnp $\delta$-doped heterojunctionbipolar transistor. Semiconductors. 43 (7), 971-974.

[7] Alexandrov, O.V., Zakhar'in, A.O., Sobolev, N.A., Shek, E.I., Makoviychuk, M.M., Parshin, E.0. 1998. Formation of donor centers after annealing of dysprosium and holmium implanted silicon. Semiconductors, 32 (9), 1029-1032.

[8] Ermolovich, I.B., Milenin, V.V., Red'ko, R.A., Red'ko, S.M. 2009. Specific features of recombination processes in CdTe films produced in different temperature conditions of growth and subsequent annealingю Semiconductors. 43 (8), 980984.

[9] Sinsermsuksakul, P. Hartman, K. Kim, S.B. Heo, J. Sun, L. Park, H.H. Chakraborty, R. Buonassisi, T. Gordon R.G. 2013. Enhancing the efficiency of SnS solar cells via bandoffset engineering with a zinc oxysulfide buffer layer. Appl. Phys. Lett., 102 (5), 053901-053905.

[10] Reynolds, J.G., Reynolds, C.L., Mohanta, Jr.A., Muth, J.F., Rowe, J.E., Everitt, H.O., Aspnes D.E. 2013. Shallow acceptor complexes in ptype ZnO. Appl. Phys. Lett. 102 (15). P. 152114-152118.

[11] Volokobinskaya, N.I., Komarov, I.N., Matyukhina, T.V., Reshetnikov, V.I., Rush, A.A., Falina, I.V., Yastrebov, A.S. 2001. A study of technological processes in the production of high-power high-voltage bipolar transistors incorporating an array of inclusions in the collector region. Semiconductors. 35 (8), 974-978.

[12] Pankratov, E.L., Bulaeva, E.A. 2013. Doping of materials during manufacture p-njunctions and bipolar transistors. Analytical approaches to model technological approaches and ways of optimization of distributions of dopants. Reviews in theoretical science. 1 (1), 58-82.

[13] Kukushkin, S.A., Osipov, A.V., Romanychev, A.I. 2016. Epitaxial growth of zinc oxide by the method of atomic layer deposition on $\mathrm{SiC} / \mathrm{Si}$ substrates. Physics of the solid state. 58 (7), 1448-1452.

[14] Trukhanov, E.M., Kolesnikov, A.V., 
Loshkarev, I.D. 2015. Long-range stresses generated by misfit dislocations in epitaxial films. Russian Microelectronics. 44 (8), 552558.

[15] Pankratov, E.L., Bulaeva, E.A. On optimization of regimes of epitaxy from gas phase. some analytical approaches to model physical processes in reactors for epitaxy from gas phase during growth films, 2015. Reviews in Theoretical Science. 3 (4), 365398.

[16] Ong, K.K. Pey, K.L. Lee, P.S. Wee, A.T.S. Wang, X.C. Chong. Y.F. 2006. Dopant distribution in the recrystallization transient at the maximum melt depth induced by laser annealing. Appl. Phys. Lett., 89 (17), 172111-172114.

[17] Wang, H.T. Tan, L.S. Chor. E.F. 2006. Pulsed laser annealing of Be-implanted GaN. J. Appl. Phys., 98 (9), 094901-094905.

[18] Bykov, Yu.V. Yeremeev, A.G. Zharova, N.A. Plotnikov, I.V. Rybakov, K.I. Drozdov, M.N. Yu.N. Drozdov, Skupov. V.D. (2003). Diffusion processes in semiconductor structures during microwave annealing. Radiophysics and Quantum Electronics, 43 (3), 836-843.

[19] Abu-Taha, J., Yazg, M. 2016. UWB distributed amplifier design using lookup tables and $g_{m}$ over $I_{D}$ methodology Analog. Integr. Circ. Sig. Process, 86, 429-438.

[20] Zhang, Y.W., Bower, A.F. (1999). Numerical simulation of island formation in a coherent strained epitaxial thin film systems. Journal of the Mechanics and Physics of Solids, 47 (11), 2273-2297.

[21] Landau, L.D., Lefshits, E.M. 2001. Theoretical physics. 7 (Theory of elasticity) (Physmatlit, Moscow).

[22] Kitayama, M., Narushima, T., Carter, W.C., Cannon, R.M., Glaeser, A.M. 2000. The Wulff shape of alumina: I, modeling the kinetics of morphological evolution. J. Am. Ceram. Soc., 83, 2561-2571; Kitayama, M., Narushima, T., Glaeser, A.M. 2000. The Wulff shape of alumina: II, experimental measurements of pore shape evolution rates. J. Am. Ceram. Soc., 83, 2572-2583.

[23] Cheremskoy, P.G., Slesov, V.V., Betekhtin, V.I. 1990. Pore in solid bodies (Energoatomizdat, Moscow).

[24] Gotra, Z.Yu. 1991. Technology of microelectronic devices (Radio and communication, Moscow).

[25] Fahey, P.M., Griffin, P.B., Plummer, J.D. 1989. Point defects and dopant diffusion in silicon. Rev. Mod. Phys., 61 (2), 289-388.
[26] Vinetskiy, V.L., Kholodar', G.A. 1979 Radiative physics of semiconductors ("Naukova Dumka", Kiev).

[27] Mynbaeva, M.G., Mokhov, E.N., Lavrent'ev, A.A., Mynbaev, K.D. 2008. High-temperature diffusion doping of porous silicon carbide. Techn. Phys. Lett. 34 (17), article № 731.

[28] Sokolov, Yu.D. 1955. About the definition of dynamic forces in the mine lifting. Applied mechanics, 1 (1), 23-35.

[29] Pankratov, E.L. 2007. Dopant diffusion dynamics and optimal diffusion time as influenced by diffusion-coefficient nonuniformity. Russian Microelectronics, 36 (1), 33-39.

[30] Pankratov, E.L. 2008. Redistribution of dopant during annealing of radiative defects in a multilayer structure by laser scans for production an implanted-junction rectifiers. Int. J. Nanoscience, 7 (4-5), 187-197.

[31] Pankratov, E.L., Bulaeva, E.A. 2013. Doping of materials during manufacture $p-n$ junctions and bipolar transistors. Analytical approaches to model technological approaches and ways of optimization of distributions of dopants.. Reviews in theoretical science, 1 (1), 58-82.

[32] Pankratov, E.L., Bulaeva, E.A. 2012. Decreasing of quantity of radiation defects in an implanted-junction rectifiers by using overlayers. Int. J. Micro-Nano Scale Transp. 3 (3), 119-130.

[33] Pankratov, E.L., Bulaeva, E.A. 2015. Optimization of manufacturing of emittercoupled logic to decrease surface of chip. International Journal of Modern Physics B. 29 (5), 1550023-1-1550023-12.

[34] Pankratov, E.L. 2017. On approach to optimize manufacturing of bipolar heterotransistors framework circuit of an operational amplifier to increase their integration rate. Influence mismatchinduced Stress. J. Comp. Theor. Nanoscience, 14 (10), 4885-4899.

[35] Pankratov, E.L., Bulaeva, E.A. 2015. An approach to increase the integration rate of planar drift heterobipolar transistors. Materials science in semiconductor processing, 34, 260-268.

[36] Pankratov, E.L., Bulaeva, E.A. 2014. An approach to manufacture of bipolar transistors in thin film structures. On the method of optimization. Int. J. Micro-Nano Scale Transp. 4 (1), 17-31.

[37] Pankratov, E.L. 2011. Increasing of the sharpness of $p$ - $n$-junctions by laser pulses. Nano, 6 (1), 31-40. 